Pacific

Journal of

Mathematics

\title{
QUANTIFYING SEPARABILITY \\ IN VIRTUALLY SPECIAL GROUPS
}

Mark F. Hagen ANd PRiYam Patel

Volume $284 \quad$ No. 1 


\title{
QUANTIFYING SEPARABILITY IN VIRTUALLY SPECIAL GROUPS
}

\author{
MARK F. HAGEN AND PRIYAM PATEL
}

\begin{abstract}
We give a new, effective proof of the separability of cubically convexcocompact subgroups of special groups. As a consequence, we show that if $G$ is a virtually compact special hyperbolic group, and $Q \leq G$ is a $K$-quasiconvex subgroup, then any $g \in G-Q$ of word length at most $n$ is separated from $Q$ by a subgroup whose index is polynomial in $n$ and exponential in $K$. This generalizes a result of Bou-Rabee and the authors on residual finiteness growth (Math. Z. 279 (2015), 297-310) and a result of Patel on surface groups (Proc. Amer. Math. Soc. 142 (2014), 2891-2906).
\end{abstract}

\section{Introduction}

Early motivation for studying residual finiteness and subgroup separability was a result of the relevance of these properties to decision problems in group theory. An observation of Dyson [1964] and Mostowski [1966], related to earlier ideas of McKinsey [1943], states that finitely presented residually finite groups have a solvable word problem. The word problem is a special case of the membership problem, i.e., the problem of determining whether a given $g \in G$ belongs to a particular subgroup $H$ of $G$. Separability can produce a solution to the membership problem in essentially the same way that a solution to the word problem is provided by residual finiteness; see, e.g., the discussion in [Aschenbrenner et al. 2015]. A subgroup $H \leq G$ is separable in $G$ if, for all $g \in G-H$, there exists $G^{\prime} \leq_{\text {f.i. }} G$ with $H \leq G^{\prime}$ and $g \notin G^{\prime}$. Producing an upper bound, in terms of $g$ and $H$, on the minimal index of such a subgroup $G^{\prime}$ is what we mean by quantifying separability of $H$ in $G$. Quantifying separability is related to the membership problem; see Remark D below.

Recently, separability has played a crucial role in low-dimensional topology, namely in the resolutions of the virtually Haken and virtually fibered conjectures [Agol 2013; Wise 2011]. Its influence in topology is a consequence of the seminal paper of Scott [1978], which establishes a topological reformulation of subgroup separability. Roughly, Scott's criterion allows one to use separability to promote

MSC2010: primary 20E26; secondary 20F36.

Keywords: subgroup separable, right-angled Artin groups, quantifying, virtually special groups. 
(appropriately construed) immersions to embeddings in finite covers. Agol [2013] proved the virtually special conjecture of Wise, an outstanding component of the proofs of the above conjectures. Agol's theorem shows that every word hyperbolic cubical group virtually embeds in a right-angled Artin group (hereafter, $R A A G$ ). Cubically convex-cocompact subgroups of RAAGs are separable [Hsu and Wise 2002; Haglund 2008] and Agol's theorem demonstrates that word hyperbolic cubical groups inherit this property via the virtual embeddings (separability properties are preserved under passing to subgroups and finite index supergroups). In fact, since quasiconvex subgroups of hyperbolic cubical groups are cubically convexcocompact [Haglund 2008; Sageev and Wise 2015], all quasiconvex subgroups of such groups are separable. In this paper, we give a new, effective proof of the separability of cubically convex-cocompact subgroups of special groups. Our main technical result is:

Theorem A. Let $\Gamma$ be a simplicial graph and let $Z$ be a compact connected cube complex, based at a 0 -cube $x$, with a based local isometry $Z \rightarrow S_{\Gamma}$. For all $g \in A_{\Gamma}-\pi_{1} Z$, there is a cube complex $(Y, x)$ such that

(1) $Z \subset Y$,

(2) there is a based local isometry $Y \rightarrow S_{\Gamma}$ such that $Z \rightarrow S_{\Gamma}$ factors as

$$
Z \hookrightarrow Y \rightarrow S_{\Gamma},
$$

(3) any closed based path representing $g$ lifts to a nonclosed path at $x$ in $Y$,

(4) $\left|Y^{(0)}\right| \leq\left|Z^{(0)}\right|(|g|+1)$,

where $|g|$ is the word length of $g$ with respect to the standard generators.

Via Haglund-Wise's canonical completion [2008], Theorem A provides the following bounds on the separability growth function (defined in Section 1) of the class of cubically convex-cocompact subgroups of a (virtually) special group. Roughly, separability growth quantifies separability of all subgroups in a given class.

Corollary B. Let $G \cong \pi_{1} X$, with $X$ a compact special cube complex, and let $\mathcal{Q}_{R}$ be the class of subgroups represented by compact local isometries to $X$ whose domains have at most $R$ vertices. Then

$$
\operatorname{Sep}_{G, \mathcal{S}}^{\mathcal{Q}_{R}}(Q, n) \leq P R n
$$

for all $Q \in \mathcal{Q}_{R}$ and $n \in \mathbb{N}$, where the constant $P$ depends only on the generating set $\mathcal{S}$. Hence, letting $\mathcal{Q}_{K}^{\prime}$ be the class of subgroups $Q \leq G$ such that the convex hull of $Q \tilde{x}$ lies in $\mathcal{N}_{K}(Q \tilde{x})$ and $\tilde{x} \in \tilde{X}^{(0)}$,

$$
\operatorname{Sep}_{G, \mathcal{S}}^{\mathcal{Q}_{K}^{\prime}}(Q, n) \leq P^{\prime} \operatorname{gr}_{\tilde{X}}(K) n,
$$

where $P^{\prime}$ depends only on $G, \tilde{X}, \mathcal{S}$, and where $\operatorname{gr}_{\tilde{X}}$ is the growth function of $\tilde{X}^{(0)}$. 
In the hyperbolic case, where cubically convex-cocompactness is equivalent to quasiconvexity, we obtain a bound that is polynomial in the length of the word and exponential in the quasiconvexity constant:

Corollary C. Let $G$ be a group with an index-J special subgroup. Fixing a word length $\|-\|_{\mathcal{S}}$ on $G$, suppose that $\left(G,\|-\|_{\mathcal{S}}\right)$ is $\delta$-hyperbolic. For each $K \geq 1$, let $\mathcal{Q}_{K}$ be the set of subgroups $Q \leq G$ such that $Q$ is $K$-quasiconvex with respect to $\|-\|_{\mathcal{S}}$. Then there exists a constant $P=P(G, \mathcal{S})$ such that for all $K \geq 0, Q \in \mathcal{Q}_{K}$, and $n \geq 0$,

$$
\operatorname{Sep}_{G, \mathcal{S}}^{\mathcal{Q}_{K}}(Q, n) \leq P \operatorname{gr}_{G}(P K)^{J !} n^{J !},
$$

where $\operatorname{gr}_{G}$ is the growth function of $G$.

Corollary $\mathrm{C}$ says that if $G$ is a hyperbolic cubical group, the subgroup $Q \leq G$ is $K$-quasiconvex, and $g \in G-Q$, then $g$ is separated from $Q$ by a subgroup of index bounded by a function polynomial in $\|g\|_{\mathcal{S}}$ and exponential in $K$.

The above results fit into a larger body of work dedicated to quantifying residual finiteness and subgroup separability of various classes of groups; see, e.g., [BouRabee and Kaletha 2012; Bou-Rabee and McReynolds 2015; Kassabov and Matucci 2011; Buskin 2009; Patel 2014; 2013; Rivin 2012; Bou-Rabee 2011; Bou-Rabee and McReynolds 2014; Kozma and Thom 2016]. When $G$ is the fundamental group of a hyperbolic surface, compare Corollary $\mathrm{C}$ to [Patel 2014, Theorem 7.1]. Combining various cubulation results with [Agol 2013], the groups covered by Corollary C include fundamental groups of hyperbolic 3-manifolds [Bergeron and Wise 2012; Kahn and Markovic 2012], hyperbolic Coxeter groups [Haglund and Wise 2010], simple-type arithmetic hyperbolic lattices [Bergeron et al. 2011], hyperbolic freeby-cyclic groups [Hagen and Wise 2015], hyperbolic ascending HNN extensions of free groups with irreducible monodromy [Hagen and Wise 2013], hyperbolic groups with a quasiconvex hierarchy [Wise 2011], $C^{\prime}\left(\frac{1}{6}\right)$ small cancellation groups [Wise 2004], and hence random groups at low enough density [Ollivier and Wise 2011], among many others.

Bou-Rabee, Hagen and Patel [2015] quantified residual finiteness for virtually special groups, by working in RAAGs and appealing to the fact that upper bounds on residual finiteness growth are inherited by finitely generated subgroups and finite index supergroups. Theorem A generalizes a main theorem of [loc. cit.], and accordingly the proof is reminiscent of the one in that reference. However, residual finiteness is equivalent to separability of the trivial subgroup, and thus it is not surprising that quantifying separability for an arbitrary convex-cocompact subgroup of a RAAG entails engagement with a more complex geometric situation. Our techniques thus significantly generalize those of [loc. cit.].

Remark D (membership problem). If $H$ is a finitely generated separable subgroup of the finitely presented group $G$, and one has an upper bound on $\operatorname{Sep}_{G, \mathcal{S}}^{\{H\}}(|g|)$ for 
some finite generating set $\mathcal{S}$ of $G$, then the following procedure decides if $g \in H$ : first, enumerate all subgroups of $G$ of index at $\operatorname{most} \operatorname{Sep}_{G, \mathcal{S}}^{\{H\}}(|g|)$ using a finite presentation of $G$. Second, for each such subgroup, test whether it contains $g$; if so, ignore it, and if not, proceed to the third step. Third, for each finite index subgroup not containing $g$, test whether it contains each of the finitely many generators of $H$; if so, we have produced a finite index subgroup containing $H$ but not $g$, whence $g \notin H$. If we exhaust the subgroups of index at $\operatorname{most} \operatorname{Sep}_{G, \mathcal{S}}^{\{H\}}(|g|)$ without finding such a subgroup, then $g \in H$. In particular, Corollary $\mathrm{C}$ gives an effective solution to the membership problem for quasiconvex subgroups of hyperbolic cubical groups, though it does not appear to be any more efficient than the more general solution to the membership problem for quasiconvex subgroups of (arbitrary) hyperbolic groups recently given by Kharlampovich, Myasnikov and Weil [Kharlampovich et al. 2014].

The paper is organized as follows. In Section 1, we define the separability growth of a group with respect to a class $\mathcal{Q}$ of subgroups, which generalizes the residual finiteness growth introduced in [Bou-Rabee 2010]. We also provide some necessary background on RAAGs and cubical geometry. In Section 2, we discuss corollaries to the main technical result, including Corollary $\mathrm{C}$, before concluding with a proof of Theorem A in Section 3.

\section{Background}

Separability growth. Let $G$ be a group generated by a finite set $\mathcal{S}$ and let $H \leq G$ be a subgroup. Let $\Omega_{H}=\{\Delta \leq G: H \leq \Delta\}$, and define a map $D_{G}^{\Omega_{H}}: G-H \rightarrow \mathbb{N} \cup\{\infty\}$ by

$$
D_{G}^{\Omega_{H}}(g)=\min \left\{[G: \Delta]: \Delta \in \Omega_{H}, g \notin \Delta\right\} .
$$

This is a special case of the notion of a divisibility function defined in [Bou-Rabee 2010] and discussed in [Bou-Rabee and McReynolds 2015]. Note that $H$ is a separable subgroup of $G$ if and only if $D_{G}^{\Omega_{H}}$ takes only finite values.

The separability growth of $G$ with respect to a class $\mathcal{Q}$ of subgroups is a function $\operatorname{Sep}_{G, \mathcal{S}}^{\mathcal{Q}}: \mathcal{Q} \times \mathbb{N} \rightarrow \mathbb{N} \cup\{\infty\}$ given by

$$
\operatorname{Sep}_{G, \mathcal{S}}^{\mathcal{Q}}(Q, n)=\max \left\{D_{G}^{\Omega_{Q}}(g): g \in G-Q,\|g\|_{\mathcal{S}} \leq n\right\} .
$$

If $\mathcal{Q}$ is a class of separable subgroups of $G$, then the separability growth measures the index of the subgroup to which one must pass in order to separate $Q$ from an element of $G-Q$ of length at most $n$. For example, when $G$ is residually finite and $\mathcal{Q}=\{\{1\}\}$, then $\operatorname{Sep}_{G, \mathcal{S}}^{\mathcal{Q}}$ is the residual finiteness growth function. The following fact is explained in greater generality in [Bou-Rabee et al. 2015, Section 2]. (In the notation of that reference, $\operatorname{Sep}_{G, \mathcal{S}}^{\mathcal{Q}}(Q, n)=\operatorname{RF}_{G, \mathcal{S}}^{\Omega_{Q}}(n)$ for all $Q \in \mathcal{Q}$ and $n \in \mathbb{N}$.) 
Proposition 1.1. Let $G$ be a finitely generated group and let $\mathcal{Q}$ be a class of subgroups of $G$. If $\mathcal{S}, \mathcal{S}^{\prime}$ are finite generating sets of $G$, then there exists a constant $C>0$ with

$$
\operatorname{Sep}_{G, \mathcal{S}^{\prime}}^{\mathcal{Q}}(Q, n) \leq C \cdot \operatorname{Sep}_{G, \mathcal{S}}^{\mathcal{Q}}(Q, C n)
$$

for $Q \in \mathcal{Q}, n \in \mathbb{N}$. Hence the asymptotic growth rate of $\operatorname{Sep}_{G, \mathcal{S}}^{\mathcal{Q}}$ is independent of $\mathcal{S}$.

(Similar statements assert that upper bounds on separability growth are inherited by finite index supergroups and arbitrary finitely generated subgroups but we do not use, and thus omit, these.)

Nonpositively curved cube complexes. We assume familiarity with nonpositively curved and CAT(0) cube complexes and refer the reader to, e.g., [Hagen 2014; Haglund 2008; Wise 2012; 2011] for background. We now make explicit some additional notions and terminology, related to convex subcomplexes, which are discussed in greater depth in [Behrstock et al. 2014]. We also discuss some basic facts about RAAGs and Salvetti complexes. Finally, we will use the method of canonical completion, introduced in [Haglund and Wise 2008], and refer the reader to [Bou-Rabee et al. 2015, Lemma 2.8] for the exact statement needed here.

Local isometries, convexity, and gates. A local isometry $\phi: Y \rightarrow X$ of cube complexes is a locally injective combinatorial map with the property that, if $e_{1}, \ldots, e_{n}$ are 1-cubes of $Y$ all incident to a 0-cube $y$, and the (necessarily distinct) 1-cubes $\phi\left(e_{1}\right), \ldots, \phi\left(e_{n}\right)$ all lie in a common $n$-cube $c$ (containing $\left.\phi(y)\right)$, then $e_{1}, \ldots, e_{n}$ span an $n$-cube $c^{\prime}$ in $Y$ with $\phi\left(c^{\prime}\right)=c$. If $\phi: Y \rightarrow X$ is a local isometry and $X$ is nonpositively curved, then $Y$ is as well. Moreover, $\phi$ lifts to an embedding $\tilde{\phi}: \tilde{Y} \rightarrow \tilde{X}$ of universal covers, and $\tilde{\phi}(\tilde{Y})$ is convex in $\tilde{X}$ in the following sense.

Let $\tilde{X}$ be a CAT( 0$)$ cube complex. The subcomplex $K \subseteq \tilde{X}$ is full if $K$ contains each $n$-cube of $\tilde{X}$ whose 1-skeleton appears in $K$. If $K$ is full, then $K$ is isometrically embedded if $K \cap \bigcap_{i} H_{i}$ is connected whenever $\left\{H_{i}\right\}$ is a set of pairwise-intersecting hyperplanes of $\tilde{X}$. Equivalently, the inclusion $K^{(1)} \hookrightarrow \tilde{X}^{(1)}$ is an isometric embedding with respect to the graph-metric. If the inclusion $K \hookrightarrow \tilde{X}$ of the full subcomplex $K$ is a local isometry, then $K$ is convex. Note that a convex subcomplex is necessarily isometrically embedded, and in fact $K$ is convex if and only if $K^{(1)}$ is metrically convex in $\tilde{X}^{(1)}$. A convex subcomplex $K$ is a CAT(0) cube complex in its own right, and its hyperplanes have the form $H \cap K$, where $K$ is a hyperplane of $\tilde{X}$. Moreover, if $K$ is convex, then hyperplanes $H_{1} \cap K, H_{2} \cap K$ of $K$ intersect if and only if $H_{1} \cap H_{2} \neq \varnothing$. We often say that the hyperplane $H$ crosses the convex subcomplex $K$ to mean that $H \cap K \neq \varnothing$ and we say the hyperplanes $H, H^{\prime}$ cross if they intersect.

Hyperplanes are an important source of convex subcomplexes, in two related ways. First, recall that for all hyperplanes $H$ of $\tilde{X}$, the carrier $\mathcal{N}(H)$ is a convex 
subcomplex. Second, $\mathcal{N}(H) \cong H \times\left[-\frac{1}{2}, \frac{1}{2}\right]$, and the subcomplexes $H \times\left\{ \pm \frac{1}{2}\right\}$ of $\tilde{X}$ "bounding" $\mathcal{N}(H)$ are convex subcomplexes isomorphic to $H$ (when $H$ is given the cubical structure in which its $n$-cubes are midcubes of $(n+1)$-cubes of $\tilde{X})$. A subcomplex of the form $H \times\left\{ \pm \frac{1}{2}\right\}$ is a combinatorial hyperplane. The convex hull of a subcomplex $\mathcal{S} \subset \tilde{X}$ is the intersection of all convex subcomplexes that contain $\mathcal{S}$; see [Haglund 2008].

Let $K \subseteq \tilde{X}$ be a convex subcomplex. Then there is a map $\mathfrak{g}_{K}: \tilde{X}^{(0)} \rightarrow K$ such that for all $x \in \tilde{X}^{(0)}$, the point $\mathfrak{g}_{K}(x)$ is the unique closest point of $K$ to $x$. (This point is often called the gate of $x$ in $K$; gates are discussed further in [Chepoi 2000; Bandelt and Chepoi 2008].) This map extends to a cubical map $\mathfrak{g}_{K}: \tilde{X} \rightarrow K$, the gate map. See, e.g., [Behrstock et al. 2014] for a detailed discussion of the gate map in the language used here; we use only that it extends the map on 0-cubes and has the property that for all $x, y$, if $\mathfrak{g}_{K}(x), \mathfrak{g}_{K}(y)$ are separated by a hyperplane $H$, then the same $H$ separates $x$ from $y$. Finally, the hyperplane $H$ separates $x$ from $\mathfrak{g}_{K}(x)$ if and only if $H$ separates $x$ from $K$. The gate map allows us to define the projection of the convex subcomplex $K^{\prime}$ of $\tilde{X}$ onto $K$ to be $\mathfrak{g}_{K^{\prime}}(K)$, which is the convex hull of the set $\left\{\mathfrak{g}_{K}(x) \in K: x \in K^{\prime(0)}\right\}$. Convex subcomplexes $K, K^{\prime}$ are parallel if $\mathfrak{g}_{K^{\prime}}(K)=K^{\prime}$ and $\mathfrak{g}_{K}\left(K^{\prime}\right)=K$. Equivalently, $K, K^{\prime}$ are parallel if and only if, for each hyperplane $H$, we have $H \cap K \neq \varnothing$ if and only if $H \cap K^{\prime} \neq \varnothing$. Note that parallel subcomplexes are isomorphic.

Remark 1.2. We often use the following facts. Let $K, K^{\prime}$ be convex subcomplexes of $\tilde{X}$. Then the convex hull $C$ of $K \cup K^{\prime}$ contains the union of $K, K^{\prime}$ and a convex subcomplex of the form $G_{K}\left(K^{\prime}\right) \times \hat{\gamma}$, where $G_{K}\left(K^{\prime}\right)$ is the image of the gate map discussed above and $\hat{\gamma}$ is the convex hull of a geodesic segment $\gamma$ joining a closest pair of 0-cubes in $K, K^{\prime}$, by [Behrstock et al. 2014, Lemma 2.4]. A hyperplane $H$ crosses $K$ and $K^{\prime}$ if and only if $H$ crosses $G_{K}\left(K^{\prime}\right)$; the hyperplane $H$ separates $K, K^{\prime}$ if and only if $H$ crosses $\hat{\gamma}$. All remaining hyperplanes either cross exactly one of $K, K^{\prime}$ or fail to cross $C$. Observe that the set of hyperplanes separating $K, K^{\prime}$ contains no triple $H, H^{\prime}, H^{\prime \prime}$ of disjoint hyperplanes, none of which separates the other two. (Such a configuration is called a facing triple.)

Salvetti complexes and special cube complexes. Let $\Gamma$ be a simplicial graph and let $A_{\Gamma}$ be the corresponding right-angled Artin group (RAAG), i.e., the group presented by

$$
\langle V(\Gamma) \mid[v, w],\{v, w\} \in E(\Gamma)\rangle,
$$

where $V(\Gamma)$ and $E(\Gamma)$ respectively denote the vertex- and edge-sets of $\Gamma$. The phrase generator of $\Gamma$ refers to this presentation; we denote each generator of $A_{\Gamma}$ by the corresponding vertex of $\Gamma$.

The RAAG $A_{\Gamma}$ is isomorphic to the fundamental group of the Salvetti complex $S_{\Gamma}$, introduced in [Charney and Davis 1995], which is a nonpositively curved cube 
complex with one 0-cube $x$, an oriented 1-cube for each $v \in V(\Gamma)$, labeled by $v$, and an $n$-torus (an $n$-cube with opposite faces identified) for every $n$-clique in $\Gamma$.

A cube complex $X$ is special if there exists a simplicial graph $\Gamma$ and a local isometry $X \rightarrow S_{\Gamma}$ inducing a monomorphism $\pi_{1} X \rightarrow A_{\Gamma}$ and a $\pi_{1} X$-equivariant embedding $\tilde{X} \rightarrow \tilde{S}_{\Gamma}$ of universal covers whose image is a convex subcomplex. Specialness allows one to study geometric features of $\pi_{1} X$ by working inside of $\tilde{S}_{\Gamma}$, which has useful structure not necessarily present in general CAT $(0)$ cube complexes; see the next section. Following Haglund and Wise [2008], a group $G$ is (virtually) [compact] special if $G$ is (virtually) isomorphic to the fundamental group of a [compact] special cube complex.

Cubical features particular to Salvetti complexes. Let $\Gamma$ be a finite simplicial graph and let $\Lambda$ be an induced subgraph of $\Gamma$. The inclusion $\Lambda \hookrightarrow \Gamma$ induces a monomorphism $A_{\Lambda} \rightarrow A_{\Gamma}$. In fact, there is an injective local isometry $S_{\Lambda} \rightarrow S_{\Gamma}$ inducing $A_{\Lambda} \rightarrow A_{\Gamma}$. Hence each conjugate $A_{\Lambda}^{g}$ of $A_{\Lambda}$ in $A_{\Gamma}$ is the stabilizer of a convex subcomplex $g \tilde{S}_{\Lambda} \subseteq \tilde{S}_{\Gamma}$. A few special cases warrant extra consideration.

When $\Lambda \subset \Gamma$ is an $n$-clique, for some $n \geq 1$, then $S_{\Lambda} \subseteq S_{\Gamma}$ is an $n$-torus, which is the Salvetti complex of the sub-RAAG isomorphic to $\mathbb{Z}^{n}$ generated by $n$ pairwisecommuting generators. In this case, $S_{\Lambda}$ is a standard $n$-torus in $S_{\Gamma}$. (When $n=1$, $S_{\Lambda}$ is a standard circle.) Each lift of $\tilde{S}_{\Lambda}$ to $\tilde{S}_{\Gamma}$ is a standard flat; when $n=1$, we use the term standard line; a compact connected subcomplex of a standard line is a standard segment. The labels and orientations of 1-cubes in $S_{\Gamma}$ pull back to $\tilde{S}_{\Gamma}$; a standard line is a convex subcomplex isometric to $\mathbb{R}$, all of whose 1-cubes have the same label, such that each 0 -cube has one incoming and one outgoing 1-cube.

When $\operatorname{Lk}(v)$ is the link of a vertex $v$ of $\Gamma$, the subcomplex $S_{\operatorname{Lk}(v)}$ is an immersed combinatorial hyperplane in the sense that $\tilde{S}_{\mathrm{Lk}(v)}$ is a combinatorial hyperplane of $\tilde{S}_{\Gamma}$. There is a corresponding hyperplane, whose carrier is bounded by $\tilde{S}_{\mathrm{Lk}(v)}$ and $v \tilde{S}_{\mathrm{Lk}(v)}$, that intersects only 1-cubes labeled by $v$. Moreover, $\tilde{S}_{\mathrm{Lk} v}$ is contained in $\tilde{S}_{\mathrm{St}(v)}$, where $\operatorname{St}(v)$ is the star of $v$, i.e., the join of $v$ and $\operatorname{Lk}(v)$. It follows that

$$
\tilde{S}_{\mathrm{St}(v)} \cong \tilde{S}_{\mathrm{Lk}(v)} \times \tilde{S}_{v}
$$

where $\tilde{S}_{v}$ is a standard line. Note that the combinatorial hyperplane $\tilde{S}_{\operatorname{Lk}(v)}$ is parallel to $v^{k} \tilde{S}_{\mathrm{Lk}(v)}$ for all $k \in \mathbb{Z}$. Likewise, $\tilde{S}_{v}$ is parallel to $g \tilde{S}_{v}$ exactly when $g \in A_{\Lambda}$, and parallel standard lines have the same labels. We say $\tilde{S}_{v}$ is a standard line dual to $\tilde{S}_{\mathrm{Lk}(v)}$, and is a standard line dual to any hyperplane $H$ such that $N(H)$ has $\tilde{S}_{\mathrm{Lk}(v)}$ as one of its bounding combinatorial hyperplanes.

Remark 1.3. We warn the reader that a given combinatorial hyperplane may correspond to distinct hyperplanes whose dual standard lines have different labels; this occurs exactly when there exist multiple vertices in $\Gamma$ whose links are the 
same subgraph. However, the standard line dual to a genuine (noncombinatorial) hyperplane is uniquely determined up to parallelism.

Definition 1.4 (frame). Let $K \subseteq \tilde{S}_{\Gamma}$ be a convex subcomplex and let $H$ be a hyperplane. Let $L$ be a standard line dual to $H$. The frame of $H$ is the convex subcomplex $H^{\prime} \times L \subseteq \tilde{S}_{\Gamma}$ described above, where $H^{\prime}$ is a combinatorial hyperplane bounding $N(H)$. If $K \subseteq \tilde{S}_{\Gamma}$ is a convex subcomplex, and $H$ intersects $K$, then the frame of $H$ in $K$ is the complex $K \cap(H \times L)$. It is shown in [Bou-Rabee et al. 2015] that the frame of $H$ in $K$ has the form $(H \cap K) \times(L \cap K)$, provided that $L$ is chosen in its parallelism class to intersect $K$. Note that the frame of $H$ is in fact well-defined, since all possible choices of $L$ are parallel.

\section{Consequences of Theorem A}

Assuming Theorem A, we quantify separability of cubically convex-cocompact subgroups of special groups with the proofs of Corollaries B and C, before proving Theorem A in the next section.

Proof of Corollary B. Let $\Gamma$ be a finite simplicial graph so that there is a local isometry $X \rightarrow S_{\Gamma}$. Let $Q \in \mathcal{Q}_{R}$ be represented by a local isometry $Z \rightarrow X$. Then for all $g \in \pi_{1} X-\pi_{1} Z$, by Theorem A, there is a local isometry $Y \rightarrow S_{\Gamma}$ such that $Y$ contains $Z$ as a locally convex subcomplex, $g \notin \pi_{1} Y$, and $\left|Y^{(0)}\right| \leq\left|Z^{(0)}\right|(|g|+1)$. Applying canonical completion [Haglund and Wise 2008] to $Y \rightarrow S_{\Gamma}$ yields a cover $\hat{S}_{\Gamma} \rightarrow S_{\Gamma}$ in which $Y$ embeds; this cover has degree $\left|Y^{(0)}\right|$ by [Bou-Rabee et al. 2015, Lemma 2.8]. Let $H^{\prime}=\pi_{1} \hat{S}_{\Gamma} \cap \pi_{1} X$, so that $\pi_{1} Z \leq H^{\prime}, g \notin H^{\prime}$, and $\left[\pi_{1} X: H^{\prime}\right] \leq\left|Z^{(0)}\right|(|g|+1)$. The first claim follows.

Let $G \cong \pi_{1} X$ with $X$ compact special, $Q \leq G$, and let the convex hull of $Q \tilde{x}$ in $\tilde{X}$ lie in $\mathcal{N}_{K}(Q \tilde{X})$. Then the second claim follows since we can choose $Z$ to be the quotient of the hull of $Q \tilde{x}$ by the action of $Q$, and $\left|Z^{(0)}\right| \leq \operatorname{gr}_{\tilde{X}}(K)$.

In general, the number of 0 -cubes in $Z$ is computable from the quasiconvexity constant of a $Q$-orbit in $\tilde{X}^{(1)}$ by [Haglund 2008, Theorem 2.28]. In the hyperbolic case, we obtain Corollary $\mathrm{C}$ in terms of the quasiconvexity constant, without reference to any particular cube complex:

Proof of Corollary $C$. We use Corollary B when $J=1$, and promote the result to a polynomial bound when $J \geq 1$. Let $Q \in \mathcal{Q}_{K}$ and let $g \in G-Q$.

The special case: Suppose $J=1$ and let $X$ be a compact special cube complex with $G \cong \pi_{1} X$. Let $Z \rightarrow X$ be a compact local isometry representing the inclusion $Q \rightarrow G$. Such a complex exists by quasiconvexity of $Q$ and [Haglund 2008, Theorem 2.28], although we shall use the slightly more computationally explicit proof in [Sageev and Wise 2015]. Let $A^{\prime} \geq 1, B^{\prime} \geq 0$ be constants such that an orbit map $\left(G,\|-\|_{\mathcal{S}}\right) \rightarrow\left(\tilde{X}^{(1)}\right.$, d) is an $\left(A^{\prime}, B^{\prime}\right)$-quasi-isometric embedding, where $\mathrm{d}$ is 
the graph-metric. Then there exist constants $A, B$, depending only on $A^{\prime}, B^{\prime}$ and hence on $\|-\|_{\mathcal{S}}$, such that $Q x$ is $(A K+B)$-quasiconvex, where $x$ is a 0 -cube in $\tilde{Z} \subset \tilde{X}$. By the proof of of [op. cit., Proposition 3.3], the convex hull $\tilde{Z}$ of $Q x$ lies in the $\rho$-neighborhood of $Q x$, where

$$
\rho=A K+B+\sqrt{\operatorname{dim} X}+\delta^{\prime}\left(\csc \left(\frac{1}{2} \sin ^{-1} \frac{1}{\sqrt{\operatorname{dim} X}}\right)+1\right)
$$

and $\delta^{\prime}=\delta^{\prime}\left(\delta, A^{\prime}, B^{\prime}\right)$. Corollary B provides $G^{\prime} \leq G$ with $g \notin G^{\prime}$, and the bound $\left[G: G^{\prime}\right] \leq\left|Z^{(0)}\right|(|g|+1)$. But $|g|+1 \leq A^{\prime}\|g\|_{\mathcal{S}}+B^{\prime}+1$, while $\left|Z^{(0)}\right| \leq \operatorname{gr}_{\tilde{X}}(\rho)$. Thus $\left[G: G^{\prime}\right] \leq \operatorname{gr}_{\tilde{X}}(\rho) A^{\prime}\|g\|_{\mathcal{S}}+\operatorname{gr}_{\tilde{X}}(\rho) B^{\prime}+\operatorname{gr}_{\tilde{X}}(\rho)$, so there exists $P_{1}$ such that

$$
\operatorname{Sep}_{G, \mathcal{S}}^{\mathcal{Q}_{K}}(Q, n) \leq P_{1} \operatorname{gr}_{\tilde{X}}\left(P_{1} K\right) n
$$

for all $K, Q \in \mathcal{Q}_{K}, n \in \mathbb{N}$, where $P_{1}$ depends only on $X$.

The virtually special case: Now suppose that $J \geq 1$. We have a compact special cube complex $X$, and $\left[G: G^{\prime}\right] \leq J$ !, where $G^{\prime} \cong \pi_{1} X$ and $G^{\prime} \triangleleft G$. Let $Q \leq G$ be a $K$-quasiconvex subgroup. By Lemma 2.1, there exists $C=C(G, \mathcal{S})$ such that $Q \cap G^{\prime}$ is $C J !(K+1)$-quasiconvex in $G$, and thus is $P_{2} C J !(K+1)$-quasiconvex in $G^{\prime}$, where $P_{2}$ depends only on $G$ and $\mathcal{S}$.

Let $g \in G-Q$. Since $G^{\prime} \triangleleft G$, the product $Q G^{\prime}$ is a subgroup of $G$ of index at most $J$ ! that contains $Q$. Hence, if $g \notin Q G^{\prime}$, then we are done. We thus assume $g \in Q G^{\prime}$. Hence we can choose a left transversal $\left\{q_{1}, \ldots, q_{s}\right\}$ for $Q \cap G^{\prime}$ in $Q$, with $s \leq J$ ! and $q_{1}=1$. Write $g=q_{i} g^{\prime}$ for some $i \leq s$, with $g^{\prime} \in G^{\prime}$. Suppose that we have chosen each $q_{i}$ to minimize $\left\|q_{i}\right\|_{\mathcal{S}}$ among all elements of $q_{i}\left(Q \cap G^{\prime}\right)$, so that, by Lemma 2.3, $\left\|q_{i}\right\| \leq J$ ! for all $i$. Hence $\left\|g^{\prime}\right\|_{\mathcal{S}} \leq\left(\|g\|_{\mathcal{S}}+J\right.$ !).

By the first part of the proof, there exists a constant $P_{1}$, depending only on $G, G^{\prime}, \mathcal{S}$, and a subgroup $G^{\prime \prime} \leq G^{\prime}$ such that $Q \cap G^{\prime} \leq G^{\prime \prime}$ and $g^{\prime} \notin G^{\prime \prime}$, and

$$
\left[G^{\prime}: G^{\prime \prime}\right] \leq P_{1} \operatorname{gr}_{G^{\prime}}\left(P_{1} P_{2} C J !(K+1)\right)\left\|g^{\prime}\right\|_{\mathcal{S}} \leq P_{1} \operatorname{gr}_{G}\left(P_{1} P_{2} C J !(K+1)\right)\left\|g^{\prime}\right\|_{\mathcal{S}} .
$$

Let $G^{\prime \prime \prime}=\bigcap_{i=1}^{s} q_{i} G^{\prime \prime} q_{i}^{-1}$, so that $g^{\prime} \notin G^{\prime \prime \prime}$ and $Q \cap G^{\prime} \leq G^{\prime \prime \prime}$ (since $G^{\prime}$ is normal), and

$$
\left[G^{\prime}: G^{\prime \prime \prime}\right] \leq\left(P_{1} \operatorname{gr}_{G}\left(P_{1} P_{2} C J !(K+1)\right)\left\|g^{\prime}\right\|_{\mathcal{S}}\right)^{s} .
$$

Finally, let $H=Q G^{\prime \prime \prime}$. This subgroup clearly contains $Q$. Suppose that $g=q_{i} g^{\prime} \in H$. Then $g^{\prime} \in Q G^{\prime \prime \prime}$, i.e., $g^{\prime}=a g^{\prime \prime \prime}$ for some $a \in Q$ and $g^{\prime \prime \prime} \in G^{\prime \prime \prime}$. Since $g^{\prime} \in G^{\prime}$ and $G^{\prime \prime \prime} \leq G^{\prime}$, we have $a \in Q \cap G^{\prime}$, whence $a \in G^{\prime \prime \prime}$, by construction. This implies that $g^{\prime} \in G^{\prime \prime \prime} \leq G^{\prime \prime}$, a contradiction. Hence $H$ is a subgroup of $G$ separating $g$ from $Q$. Finally,

$$
[G: H] \leq\left[G: G^{\prime \prime \prime}\right] \leq J !\left[P_{1} \operatorname{gr}_{G}\left(P_{1} P_{2} C J !(K+1)\right)\left(\|g\|_{\mathcal{S}}+J !\right)\right]^{J !},
$$

and the proof is complete. 
Lemma 2.1. Let the group $G$ be generated by a finite set $\mathcal{S}$ and let $\left(G,\|-\|_{\mathcal{S}}\right)$ be $\delta$-hyperbolic. Let $Q \leq G$ be $K$-quasiconvex, and let $G^{\prime} \leq G$ be an index-I subgroup. Then $Q \cap G^{\prime}$ is $C I(K+1)$-quasiconvex in $G$ for some $C$ depending only on $\delta$ and $\mathcal{S}$.

Proof. Since $Q$ is $K$-quasiconvex in $G$, it is generated by a set $\mathcal{T}$ of $q \in Q$ with $\|q\|_{\mathcal{S}} \leq 2 K+1$ by [Bridson and Haefliger 1999, Lemma III.Г.3.5]. A standard argument shows $\left(Q,\|-\|_{\mathcal{T}}\right) \hookrightarrow\left(G,\|-\|_{\mathcal{S}}\right)$ is a $(2 K+1,0)$-quasi-isometric embedding. Lemma 2.3 shows that $Q \cap G^{\prime}$ is $I$-quasiconvex in $\left(Q,\|-\|_{\mathcal{T}}\right)$, since $\left[Q: Q \cap G^{\prime}\right] \leq I$. Hence $Q \cap G^{\prime}$ has a generating set making it $((2 I+1)(2 K+1), 0)$ quasi-isometrically embedded in $\left(G,\|-\|_{\mathcal{S}}\right)$. Apply Lemma 2.2 to conclude.

The next lemma is standard, but we include it to highlight the constants involved:

Lemma 2.2. Let $G$ be a group generated by a finite set $\mathcal{S}$ and suppose that $\left(G,\|-\|_{\mathcal{S}}\right)$ is $\delta$-hyperbolic. Then there exists a (sub)linear function $f: \mathbb{N} \rightarrow \mathbb{N}$, depending on $\mathcal{S}$ and $\delta$, such that $\sigma \subseteq \mathcal{N}_{f(\lambda)}(\gamma)$ whenever $\gamma:[0, L] \rightarrow G$ is a $(\lambda, 0)$-quasigeodesic and $\sigma$ is a geodesic joining $\gamma(0)$ to $\gamma(L)$.

Proof. See, e.g., the proof of [Bridson and Haefliger 1999, Theorem III.H.1.7].

Lemma 2.3. Let $Q$ be a group generated by a finite set $\mathcal{S}$ and let $Q^{\prime} \leq Q$ be a subgroup with $\left[Q: Q^{\prime}\right]=s<\infty$. Then there exists a left transversal $\left\{q_{1}, \ldots, q_{s}\right\}$ for $Q^{\prime}$ such that $\left\|q_{i}\right\|_{\mathcal{S}} \leq s$ for $1 \leq i \leq s$. Hence $Q^{\prime}$ is $s$-quasiconvex in $Q$.

Proof. Suppose that $q_{k}=s_{i_{k}} \cdots s_{i_{1}}$ is a geodesic word in $\mathcal{S} \cup \mathcal{S}^{-1}$ and that $q_{k}$ is a shortest representative of $q_{k} Q^{\prime}$. Let $q_{j}=s_{i_{j}} \cdots s_{i_{1}}$ be the word in $Q$ consisting of the last $j$ letters of $q_{k}$ for all $1<j<k$, and let $q_{1}=1$. We claim that each $q_{j}$ is a shortest representative for $q_{j} Q^{\prime}$. Otherwise, there would exist $p$ with $\|p\|_{\mathcal{S}}<j$ such that $q_{j} Q^{\prime}=p Q^{\prime}$. But then $s_{k} \cdots s_{j+1} p Q^{\prime}=q_{k} Q^{\prime}$, and thus $q_{k}$ was not a shortest representative. It also follows immediately that $q_{j} Q^{\prime} \neq q_{j^{\prime}} Q^{\prime}$ for $j \neq j^{\prime}$. Thus, $q_{1}, q_{2}, \ldots, q_{k}$ represent distinct left cosets of $Q^{\prime}$ provided $k \leq s$, and the claim follows.

Remark 2.4 (embeddings in finite covers). Given a compact special cube complex $X$ and a compact local isometry $Z \rightarrow X$, Theorem A gives an upper bound on the minimal degree of a finite cover in which $Z$ embeds; indeed, producing such an embedding entails separating $\pi_{1} Z$ from finitely many elements in $\pi_{1} X$. However, it is observed in [Bou-Rabee et al. 2015, Lemma 2.8] that the Haglund-Wise canonical completion construction [2008] produces a cover $\hat{X} \rightarrow X$ of degree $\left|Z^{(0)}\right|$ in which $Z$ embeds.

\section{Proof of Theorem A}

In this section, we give a proof of the main technical result.

Definition 3.1. Let $S_{\Gamma}$ be a Salvetti complex and let $\tilde{S}_{\Gamma}$ be its universal cover. The hyperplanes $H, H^{\prime}$ of $\tilde{S}_{\Gamma}$ are collateral if they have a common dual standard line 
(equivalently, the same frame). Clearly collateralism is an equivalence relation, and collateral hyperplanes are isomorphic and have the same stabilizer.

Being collateral implies that the combinatorial hyperplanes bounding the carrier of $H$ are parallel to those bounding the carrier of $H^{\prime}$. However, the converse is not true when $\Gamma$ contains multiple vertices whose links coincide. In the proof of Theorem A, we will always work with hyperplanes, rather than combinatorial hyperplanes, unless we explicitly state otherwise.

Proof of Theorem A. Let $\tilde{x} \in \tilde{S}_{\Gamma}$ be a lift of the base 0-cube $x$ in $S_{\Gamma}$, and let $\tilde{Z} \subseteq \tilde{S}_{\Gamma}$ be the lift of the universal cover of $Z$ containing $\tilde{x}$. Since $Z \rightarrow S_{\Gamma}$ is a local isometry, $\tilde{Z}$ is convex. Let $\hat{Z} \subset \tilde{Z}$ be the convex hull of a compact connected fundamental domain for the action of $\pi_{1} Z \leq A_{\Gamma}$ on $\tilde{Z}$. Denote by $K$ the convex hull of $\hat{Z} \cup\{g \tilde{x}\}$ and let $\mathfrak{S}$ be the set of hyperplanes of $\tilde{S}_{\Gamma}$ intersecting $K$. We will form a quotient of $K$, restricting to $\hat{Z} \rightarrow Z$ on $\hat{Z}$, whose image admits a local isometry to $S_{\Gamma}$.

The subcomplex $\lfloor\hat{Z}\rfloor$. Let $\mathfrak{L}$ be the collection of standard segments $\ell$ in $K$ that map to standard circles in $S_{\Gamma}$ with the property that $\ell \cap \hat{Z}$ has noncontractible image in $Z$. Let $\lfloor\hat{Z}\rfloor$ be the convex hull of $\hat{Z} \cup \bigcup_{\ell \in \mathfrak{L}} \ell$, so that $\hat{Z} \subseteq\lfloor\hat{Z}\rfloor \subseteq K$.

Partitioning $\mathfrak{S}$. We now partition $\mathfrak{S}$ according to the various types of frames in $K$. First, let $\mathfrak{Z}$ be the set of hyperplanes intersecting $\hat{Z}$. Second, let $\mathfrak{N}$ be the set of $N \in \mathfrak{S}-\mathfrak{Z}$ such that the frame $(N \cap K) \times(L \cap K)$ of $N$ in $K$ has the property that for some choice of $x_{0} \in N^{(0)}$, the segment $\left(\left\{x_{0}\right\} \times L\right) \cap \hat{Z}$ maps to a nontrivial cycle of 1 -cubes in $Z$. Let $n_{N} \geq 1$ be the length of that cycle. By convexity of $\hat{Z}$, the number $n_{N}$ is independent of the choice of the segment $L$ within its parallelism class. Note that $\mathfrak{N}$ is the set of hyperplanes that cross $\lfloor\hat{Z}\rfloor$, but do not cross $\hat{Z}$. Hence each $N \in \mathfrak{N}$ is collateral to some $W \in \mathfrak{Z}$. Third, fix a collection $\left\{H_{1}, \ldots H_{k}\right\} \subset \mathfrak{S}-\mathfrak{Z}$ such that:

(1) For $1 \leq i \leq k-1$, the hyperplane $H_{i}$ separates $H_{i+1}$ from $\lfloor\hat{Z}\rfloor$.

(2) For $1 \leq i<j \leq k$, if a hyperplane $H$ separates $H_{i}$ from $H_{j}$, then $H$ is collateral to $H_{\ell}$ for some $\ell \in[i, j]$. Similarly, if $H$ separates $H_{1}$ from $\lfloor\hat{Z}\rfloor$, then $H$ is collateral to $H_{1}$, and if $H$ separates $H_{k}$ from $g \tilde{x}$, then $H$ is collateral to $H_{k}$.

(3) For each $i$, the frame $\left(H_{i} \cap K\right) \times L_{i}$ of $H_{i}$ in $K$ has the property that for every $h \in H_{i}^{(0)}$, the image in $Z$ of the segment $\left(\{h\} \times L_{i}\right) \cap \hat{Z}$ is empty or contractible. (Here, $L_{i}$ is a standard segment of a standard line dual to $H_{i}$.)

Let $\mathfrak{H}$ be the set of all hyperplanes of $\mathfrak{S}-\mathfrak{Z}$ that are collateral to $H_{i}$ for some $i$. Condition (3) above ensures that $\mathfrak{H} \cap \mathfrak{N}=\varnothing$, while $\mathfrak{H}=\varnothing$ only if $K=\lfloor\hat{Z}\rfloor$. Finally, let $\mathfrak{B}=\mathfrak{S}-(\mathfrak{Z} \cup \mathfrak{N} \cup \mathfrak{H})$. Note that each $B \in \mathfrak{B}$ crosses some $H_{i}$. Figure 1 shows a possible $K$ and various families of hyperplanes crossing it. 


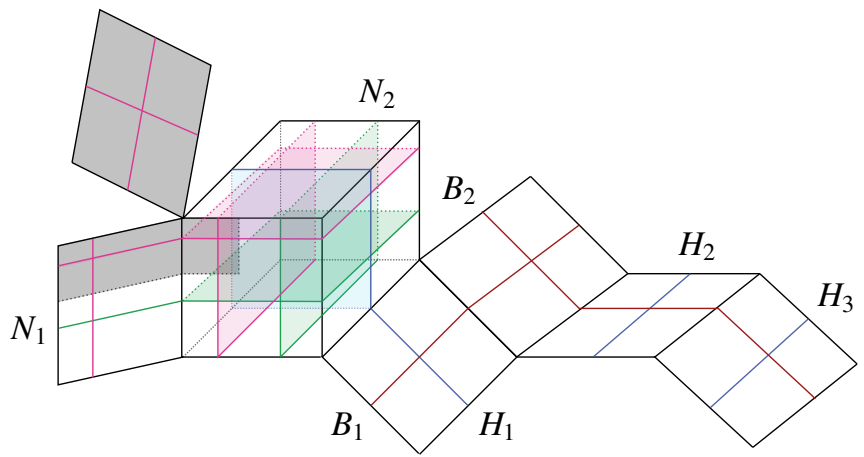

Figure 1. Hyperplanes crossing $K$ (the dark shaded area on the left is $\hat{Z}$ ).

Mapping $\lfloor\hat{Z}\rfloor$ to $Z$. We now define a quotient map $\mathfrak{q}:\lfloor\hat{Z}\rfloor \rightarrow Z$ extending the restriction $\hat{Z} \rightarrow Z$ of $\tilde{Z} \rightarrow Z$. Note that if $\mathfrak{N}=\varnothing$, then $\lfloor\hat{Z}\rfloor=\hat{Z}$, and $\mathfrak{q}$ is just the map $\hat{Z} \rightarrow Z$. Hence suppose $\mathfrak{N} \neq \varnothing$ and let $\mathfrak{N}_{1}, \ldots, \mathfrak{N}_{s}$ be the collateralism classes of hyperplanes in $\mathfrak{N}$, and for $1 \leq i \leq s$, let $\mathfrak{N}_{i}^{\prime}$ be the collateralism class of $\mathfrak{N}_{i}$ in $\mathfrak{S}$, i.e., $\mathfrak{N}_{i}$ together with a nonempty set of collateral hyperplanes in $\mathfrak{Z}$. For each $i$, let $L_{i}$ be a maximal standard line segment of $\lfloor\hat{Z}\rfloor$, each of whose 1-cubes is dual to a hyperplane in $\mathfrak{N}_{i}^{\prime}$ and which crosses each element of $\mathfrak{N}_{i}^{\prime}$. For each $i$, let $N_{i} \in \mathfrak{N}_{i}$ be a hyperplane separating $\hat{Z}$ from $g \tilde{x}$. Then $N_{i} \cap N_{j} \neq \varnothing$ for $i \neq j$, since neither separates the other from $\hat{Z}$. We can choose the $L_{i}$ so that there is an isometric embedding $\prod_{i=1}^{k} L_{i} \rightarrow\lfloor\hat{Z}\rfloor$, since whether or not two hyperplanes of $\tilde{S}_{\Gamma}$ cross depends only on their collateralism classes.

For each nonempty $I \subseteq\{1, \ldots, k\}$, a hyperplane $W \in \mathfrak{Z}$ crosses some $U \in \bigcup_{i \in I} \mathfrak{N}_{i}^{\prime}$ if and only if $W$ crosses each hyperplane collateral to $U$. Hence, by Lemma 7.11 of [Hagen 2014], there is a maximal convex subcomplex $Y(I) \subset \hat{Z}$, defined up to parallelism, such that a hyperplane $W$ crosses each $U \in \bigcup_{i \in I} \mathfrak{N}_{i}^{\prime}$ if and only if $W \cap Y(I) \neq \varnothing$. Let $\mathfrak{A}(I)$ be the set of hyperplanes crossing $Y(I)$. By the definition of $Y(I)$ and the lemma just cited, there is a combinatorial isometric embedding $Y(I) \times \prod_{i \in I} L_{i} \rightarrow\lfloor\hat{Z}\rfloor$, whose image we denote by $F(I)$ and refer to as a generalized frame. Moreover, for any 0 -cube $z \in\lfloor\hat{Z}\rfloor$ that is not separated from a hyperplane in $\bigcup_{i \in I} \mathfrak{N}_{i}^{\prime} \cup \mathfrak{A}(I)$ by a hyperplane not in that set, we can choose $F(I)$ to contain $z$; this follows from the proof of the same lemma of Hagen. Figures 2 and 3 show possible collateralism classes $\mathfrak{N}_{i}^{\prime}$ and generalized hyperplane frames.

To build $\mathfrak{q}$, we will express $\lfloor\hat{Z}\rfloor$ as the union of $\hat{Z}$ and a collection of generalized frames, define $\mathfrak{q}$ on each generalized frame, and check that the definition is compatible where multiple generalized frames intersect. Let $z \in\lfloor\hat{Z}\rfloor$ be a 0 -cube. Either $z \in \hat{Z}$, or there is a nonempty set $I \subset\{1, \ldots, k\}$ such that the set of hyperplanes separating $z$ from $\hat{Z}$ is contained in $\bigcup_{i \in I} \mathfrak{N}_{i}^{\prime}$, and each $\mathfrak{N}_{i}^{\prime}$ contains a hyperplane 

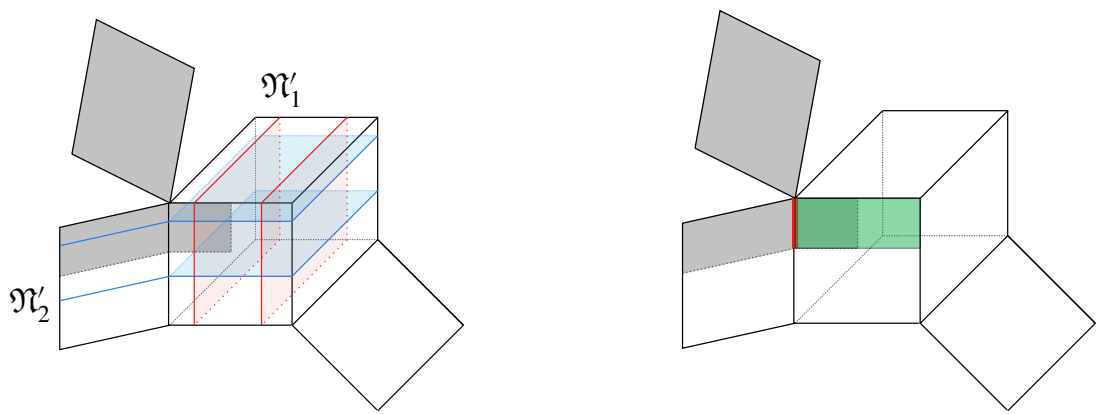

Figure 2. Collateral families $\mathfrak{N}_{1}^{\prime}$ and $\mathfrak{N}_{2}^{\prime}$ (left) and $Y(\{1\}) \times L_{1}$ (right).
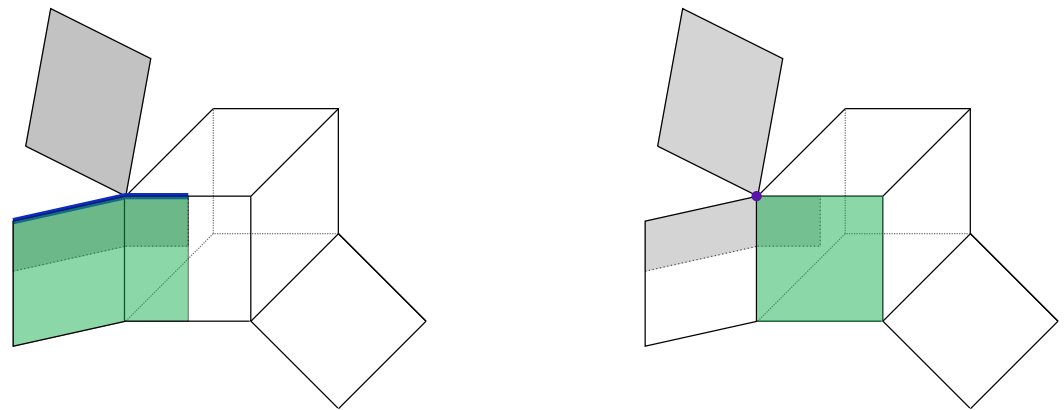

Figure 3. $Y(\{2\}) \times L_{2}$ (left) and $Y(\{1,2\}) \times\left(L_{1} \times L_{2}\right)($ right $)$.

separating $z$ from $\hat{Z}$. If $H \in \mathfrak{A}(I) \cup \bigcup_{i \in I} \mathfrak{N}_{i}^{\prime}$ is separated from $z$ by a hyperplane $U$, then $U \in \mathfrak{A}(I) \cup \bigcup_{i \in I} \mathfrak{N}_{i}^{\prime}$, whence we can choose $F(I)$ to contain $z$. Hence $\lfloor\hat{Z}\rfloor$ is the union of $\hat{Z}$ and a finite collection of generalized frames $F\left(I_{1}\right), \ldots, F\left(I_{t}\right)$.

For any $p \in\{1, \ldots, t\}$, we have $F\left(I_{p}\right)=Y\left(I_{p}\right) \times \prod_{j \in I_{p}} L_{j}$ and we define $\bar{Y}\left(I_{p}\right)=\operatorname{im}\left(Y\left(I_{p}\right) \rightarrow Z\right)$. Also, let $\bar{L}_{j}=\operatorname{im}\left(L_{j} \cap \hat{Z} \rightarrow Z\right)$ be the cycle of length $n_{N_{j}}$ to which $L_{j}$ maps, for each $j \in I_{p}$. Note that $Z$ contains $\bar{F}\left(I_{p}\right)=\bar{Y}\left(I_{p}\right) \times \prod_{j \in I_{P}} \bar{L}_{j}$ and so we define the quotient map $\mathfrak{q}_{p}: F\left(I_{p}\right) \rightarrow Z$ as the product of the above combinatorial quotient maps, namely, $\mathfrak{q}_{p}\left(y,\left(r_{j}\right)_{j \in I_{P}}\right)=\left(\bar{y},\left(r_{j} \bmod n_{N_{j}}\right)_{j \in I_{p}}\right)$ for $y \in Y\left(I_{p}\right)$ and $r_{j} \in L_{j}$.

To ensure that $\mathfrak{q}_{p}\left(F\left(I_{p}\right) \cap F\left(I_{j}\right)\right)=\mathfrak{q}_{j}\left(F\left(I_{p}\right) \cap F\left(I_{j}\right)\right)$ for all $i, j \leq t$, it suffices to show that

$$
F\left(I_{p}\right) \cap F\left(I_{j}\right):=\left(Y\left(I_{p}\right) \times \prod_{k \in I_{p}} L_{k}\right) \cap\left(Y\left(I_{j}\right) \times \prod_{\ell \in I_{j}} L_{\ell}\right)=\left[Y\left(I_{p}\right) \cap Y\left(I_{j}\right)\right] \times \prod_{k \in I_{p} \cap I_{j}} L_{k} .
$$

This in turn follows from [Caprace and Sageev 2011, Proposition 2.5]. Hence, the quotient maps $\mathfrak{q}_{p}$ are compatible and thus define a combinatorial quotient map $\mathfrak{q}:\lfloor\hat{Z}\rfloor \rightarrow Z$ extending the maps $\mathfrak{q}_{p}$. 
Observe that if $\mathfrak{H}=\varnothing$, i.e., $K=\lfloor\hat{Z}\rfloor$, then we take $Y=Z$. By hypothesis, $Z$ admits a local isometry to $S_{\Gamma}$ and has the desired cardinality. Moreover, our hypothesis on $g$ ensures that $g \notin \pi_{1} Y$, but the map $\mathfrak{q}$ shows that any closed combinatorial path in $S_{\Gamma}$ representing $g$ lifts to a (nonclosed) path in $Z$, so the proof of the theorem is complete. Thus we can and shall assume that $\mathfrak{H} \neq \varnothing$.

Quotients of $\mathfrak{H}$-frames. To extend $\mathfrak{q}$ to the rest of $K$, we now describe quotient maps, compatible with the map $\hat{Z} \rightarrow Z$, on frames associated to hyperplanes in $\mathfrak{H}$. An isolated $\mathfrak{H}$-frame is a frame $(H \cap K) \times L$, where $H \in \mathfrak{H}$ and $H$ crosses no hyperplane of $\hat{Z}$ (and hence crosses no hyperplane of $\lfloor\hat{Z}\rfloor$ ). An interfered $\mathfrak{H}$-frame is a frame $(H \cap K) \times L$, where $H \in \mathfrak{H}$ and $H$ crosses an element of $\mathfrak{Z}$. Equivalently, $(H \cap K) \times L$ is interfered if $\mathfrak{g}_{N(H)}(\hat{Z})$ contains a 1-cube and is isolated otherwise.

Define quotient maps on isolated $\mathfrak{H}$-frames by the same means as was used for arbitrary frames in [Bou-Rabee et al. 2015]. Let $(H \cap K) \times L$ be an isolated $\mathfrak{H}$-frame. Let $\bar{H}$ be the immersed hyperplane in $S_{\Gamma}$ to which $H$ is sent by $\tilde{S}_{\Gamma} \rightarrow S_{\Gamma}$, and let $\overline{H \cap K}$ be the image of $H \cap K$. We form a quotient $Y_{H}=\overline{H \cap K} \times L$ of every isolated $\mathfrak{H}$-frame $(H \cap K) \times L$.

Now we define the quotients of interfered $\mathfrak{H}$-frames. Let $\hat{A}=\mathfrak{g}_{N(H)}(\hat{Z})$ and let $A$ be the image of $\hat{A}$ under $\hat{Z} \rightarrow Z$. There is a local isometry $A \rightarrow S_{\Gamma}$, to which we apply canonical completion to produce a finite cover $\dddot{S}_{\Gamma} \rightarrow S_{\Gamma}$ where $A$ embeds. By [Bou-Rabee et al. 2015, Lemma 2.8], $\operatorname{deg}\left(\dddot{S}_{\Gamma} \rightarrow S_{\Gamma}\right)=\left|\dddot{S}_{\Gamma}^{(0)}\right|=\left|A^{(0)}\right| \leq\left|Z^{(0)}\right|$. Let $\overline{H \cap K}=\operatorname{im}\left(H \cap K \rightarrow \dddot{S}_{\Gamma}\right)$, and map the interfered $\mathfrak{H}$-frame $(H \cap K) \times L$ to $Y_{H}=\overline{H \cap K} \times L$.

Constructing $Y$. We now construct a compact cube complex $Y^{\prime}$ from $Z$ and the various quotients $Y_{H}$. A hyperplane $W$ in $K$ separates $H_{1}$ from $\hat{Z}$ only if $W \in \mathfrak{N}$. Each $\mathfrak{H}$-hyperplane frame has the form $\left(H_{i} \cap K\right) \times L_{i}=\left(H_{i} \cap K\right) \times\left[0, m_{i}\right]$, parametrized so that $\left(H_{i} \cap K\right) \times\{0\}$ is the closest combinatorial hyperplane in the frame to $\hat{Z}$. We form $Y^{\prime}(1)$ by gluing $Y_{H_{1}}$ to $Z$ along the image of $\mathfrak{g}_{\hat{Z}}\left(\left(H_{1} \cap K\right) \times\{0\}\right)$, enabled by the fact that the quotients of interfered $\mathfrak{H}$-frames are compatible with $\hat{Z} \rightarrow Z$. In a similar manner, form $Y^{\prime}(i)$ from $Y^{\prime}(i-1)$ and $Y_{H_{i}}$ by identifying the image of $\left(H_{i-1} \cap K\right) \times\left\{m_{i-1}\right\} \cap\left(H_{i} \cap K\right) \times\{0\}$ in $Y_{H_{i-1}} \subset Y^{\prime}(i-1)$ with its image in $Y_{H_{i}}$. Let $Y^{\prime}=Y^{\prime}(k)$.

Let

$$
K^{\prime}=\lfloor\hat{Z}\rfloor \cup \bigcup_{H_{i} \in \mathfrak{H}}\left(H_{i} \cap K\right) \times L_{i} .
$$

Since $H_{i} \cap H_{j}=\varnothing$ for $i \neq j$, there exists a map $\left(K^{\prime}, \tilde{x}\right) \rightarrow\left(Y^{\prime}, x\right)$ and a map $\left(Y^{\prime}, x\right) \rightarrow\left(S_{\Gamma}, x\right)$ such that the composition is precisely the restriction to $K^{\prime}$ of the covering map $\left(\tilde{S}_{\Gamma}, \tilde{x}\right) \rightarrow\left(S_{\Gamma}, x\right)$.

If $Y^{\prime} \rightarrow S_{\Gamma}$ fails to be a local isometry, then there exists $i$ and nontrivial open cubes $e \subset \overline{H_{i-1} \cap K} \times\left\{m_{i-1}\right\}$ (or $Z$ if $i=1$ ) and $c \subset \overline{H_{i} \cap K} \times\{0\}$ such that $S_{\Gamma}$ 
contains an open cube $\bar{e} \times \bar{c}$, where $\bar{e}, \bar{c}$ are the images of $e, c$ under $\tilde{S}_{\Gamma} \rightarrow S_{\Gamma}$, respectively. Moreover, since $\mathfrak{g}_{\hat{Z}}\left(H_{i} \cap K\right) \subseteq \mathfrak{g}_{\hat{Z}}\left(H_{i-1} \cap K\right)$, we can assume that $\bar{c}$ is disjoint from each immersed hyperplane of $S_{\Gamma}$ crossing $Z$. Hence the closure $C l(\bar{c})$ is a standard torus. Glue $C l(\bar{e}) \times C l(\bar{c})$ to $Y^{\prime}$, if necessary, in the obvious way. Note that this gluing adds no new 0 -cubes to $Y^{\prime}$. Indeed, every 0 -cube of $C l(\bar{e}) \times C l(\bar{c})$ is identified with an existing 0-cube of $Y^{\prime}$ lying in $\overline{H_{i-1} \cap K} \times\left\{m_{i-1}\right\}$. Adding $C l(\bar{e}) \times C l(\bar{c})$ also preserves the existence of a local injection from our cube complex to $S_{\Gamma}$. Either this new complex admits a local isometry to $S_{\Gamma}$, or there is a missing cube of the form $\bar{e} \times \bar{c}$ where $C l(\bar{c})$ is a standard torus and $\bar{e}$ lies in $Y^{\prime}$. We add cubes of this type until we have no missing corners. That the process terminates in a local isometry with compact domain $Y$ is a consequence of the following facts: at each stage, every missing cube has the form $\bar{e} \times \bar{c}$ where $\bar{e}$ lies in $Y^{\prime}$ and $C l(\bar{c})$ is a standard torus, so the number of 0-cubes remains unchanged; each gluing preserves the existence of a local injection to $S_{\Gamma}$; each gluing increases the number of positive dimensional cubes containing some 0 -cube; cubes that we add are images of cubes in $K$, which is compact.

There exists a combinatorial path $\gamma$ in $K^{\prime}$ joining $\tilde{x}$ to $g \tilde{x}$. It follows from the existence of $\gamma$ that the convex hull of $K^{\prime}$ is precisely equal to $K$. Hence, there exists a based cubical map $(K, \tilde{x}) \rightarrow(Y, x) \rightarrow\left(S_{\Gamma}, x\right)$, so that the composition is the restriction of the covering map $\left(\tilde{S}_{\Gamma}, \tilde{x}\right) \rightarrow\left(S_{\Gamma}, x\right)$. Therefore, any closed path in $S_{\Gamma}$ representing $g$ lifts to a nonclosed path at $x$ in $Y$. It is easily verified that the number of 0 -cubes in $Y$ is bounded by $\left|Z^{(0)}\right|\left(m_{1}+\cdots+m_{k}\right)$, where each $m_{i}$ is the length of $L_{i}$, and hence $\left|Y^{(0)}\right| \leq\left|Z^{(0)}\right|(|g|+1)$. Thus, $Y$ is the desired cube complex.

Remark 3.2. When $\operatorname{dim} S_{\Gamma}=1$, arguing as above shows that $Y$ can be chosen so that $\left|Y^{(0)}\right| \leq\left|Z^{(0)}\right|+|g|$. Hence, if $F$ is freely generated by $\mathcal{S}$, with $|\mathcal{S}|=r$, then $\operatorname{Sep}_{F, \mathcal{S}}^{\mathcal{Q}_{K}}(Q, n) \leq(2 r)^{K}+n$.

\section{Acknowledgments}

We thank K. Bou-Rabee, S. Dowdall, F. Haglund, D.B. McReynolds, N. Miller, H. Wilton, and D.T. Wise for helpful discussions about issues related to this paper. We also thank an anonymous referee for astute corrections. The authors acknowledge travel support from U.S. National Science Foundation grants DMS 1107452, 1107263, 1107367 "RNMS: Geometric Structures and Representation Varieties" (the GEAR Network) and from grant NSF 1045119. Hagen was supported by the National Science Foundation under Grant Number NSF 1045119.

\section{References}

[Agol 2013] I. Agol, “The virtual Haken conjecture”, Doc. Math. 18 (2013), 1045-1087. MR 3104553 Zbl 1286.57019 
[Aschenbrenner et al. 2015] M. Aschenbrenner, S. Friedl, and H. Wilton, "Decision problems for 3manifolds and their fundamental groups", Geom. Topol. Monogr. 19 (2015), 201-236. Zbl 06537400

[Bandelt and Chepoi 2008] H.-J. Bandelt and V. Chepoi, "Metric graph theory and geometry: a survey", pp. 49-86 in Surveys on discrete and computational geometry, edited by J. E. Goodman et al., Contemporary Mathematics 453, American Mathematical Society, Providence, RI, 2008. MR 2405677 Zbl 1169.05015

[Behrstock et al. 2014] J. Behrstock, M. F. Hagen, and A. Sisto, "Hierarchically hyperbolic spaces, I: Curve complexes for cubical groups", preprint, 2014. arXiv 1412.2171

[Bergeron and Wise 2012] N. Bergeron and D. T. Wise, "A boundary criterion for cubulation", Amer. J. Math. 134:3 (2012), 843-859. MR 2931226 Zbl 1279.20051

[Bergeron et al. 2011] N. Bergeron, F. Haglund, and D. T. Wise, "Hyperplane sections in arithmetic hyperbolic manifolds", J. Lond. Math. Soc. (2) 83:2 (2011), 431-448. MR 2776645 Zbl 1236.57021

[Bou-Rabee 2010] K. Bou-Rabee, "Quantifying residual finiteness", J. Algebra 323:3 (2010), 729737. MR 2574859 Zbl 1222.20020

[Bou-Rabee 2011] K. Bou-Rabee, "Approximating a group by its solvable quotients", New York J. Math. 17 (2011), 699-712. MR 2851069 Zbl 1243.20038

[Bou-Rabee and Kaletha 2012] K. Bou-Rabee and T. Kaletha, "Quantifying residual finiteness of arithmetic groups”, Compos. Math. 148:3 (2012), 907-920. MR 2925403 Zbl 1256.20030

[Bou-Rabee and McReynolds 2014] K. Bou-Rabee and D. B. McReynolds, "Characterizing linear groups in terms of growth properties", preprint, 2014. To appear in Mich. Math. J. arXiv 1403.0983

[Bou-Rabee and McReynolds 2015] K. Bou-Rabee and D. B. McReynolds, "Extremal behavior of divisibility functions”, Geom. Dedicata 175 (2015), 407-415. MR 3323650 Zbl 1314.20023

[Bou-Rabee et al. 2015] K. Bou-Rabee, M. F. Hagen, and P. Patel, "Residual finiteness growths of virtually special groups", Math. Z. 279:1-2 (2015), 297-310. MR 3299854 Zbl 1317.20036

[Bridson and Haefliger 1999] M. R. Bridson and A. Haefliger, Metric spaces of non-positive curvature, Grundlehren der Mathematischen Wissenschaften 319, Springer, Berlin, 1999. MR 1744486 Zbl 0988.53001

[Buskin 2009] N. V. Buskin, “Экономная отделимость в свободных группах”, Sibirsk. Mat. Zh. 50:4 (2009), 765-771. Translated as "Economical separability in free groups" in Siberian Math. J. 50:4 (2009), 603-608. MR 2583614 Zbl 1212.20055

[Caprace and Sageev 2011] P.-E. Caprace and M. Sageev, "Rank rigidity for CAT(0) cube complexes", Geom. Funct. Anal. 21:4 (2011), 851-891. MR 2827012 Zbl 1266.20054

[Charney and Davis 1995] R. Charney and M. W. Davis, "Finite $K(\pi, 1)$ 's for Artin groups", pp. 110-124 in Prospects in topology (Princeton, NJ, 1994), edited by F. Quinn, Annals of Mathematics Studies 138, Princeton University Press, 1995. MR 1368655 Zbl 0930.55006

[Chepoi 2000] V. Chepoi, "Graphs of some CAT(0) complexes", Adv. in Appl. Math. 24:2 (2000), 125-179. MR 1748966 Zbl 1019.57001

[Dyson 1964] V. Dyson, "The word problem and residually finite groups", Not. Amer. Math. Soc. 11 (1964), 743.

[Hagen 2014] M. F. Hagen, "Weak hyperbolicity of cube complexes and quasi-arboreal groups", $J$. Topol. 7:2 (2014), 385-418. MR 3217625 Zbl 06366501

[Hagen and Wise 2013] M. F. Hagen and D. T. Wise, "Cubulating hyperbolic free-by-cyclic groups: the irreducible case", preprint, 2013. arXiv 1311.2084 
[Hagen and Wise 2015] M. F. Hagen and D. T. Wise, "Cubulating hyperbolic free-by-cyclic groups: the general case”, Geom. Funct. Anal. 25:1 (2015), 134-179. MR 3320891 Zbl 06422799

[Haglund 2008] F. Haglund, "Finite index subgroups of graph products", Geom. Dedicata 135 (2008), 167-209. MR 2413337 Zbl 1195.20047

[Haglund and Wise 2008] F. Haglund and D. T. Wise, "Special cube complexes", Geom. Funct. Anal. 17:5 (2008), 1551-1620. MR 2377497 Zbl 1155.53025

[Haglund and Wise 2010] F. Haglund and D. T. Wise, "Coxeter groups are virtually special", Adv. Math. 224:5 (2010), 1890-1903. MR 2646113 Zbl 1195.53055

[Hsu and Wise 2002] T. Hsu and D. T. Wise, "Separating quasiconvex subgroups of right-angled Artin groups", Math. Z. 240:3 (2002), 521-548. MR 1924020 Zbl 1006.20028

[Kahn and Markovic 2012] J. Kahn and V. Markovic, "Immersing almost geodesic surfaces in a closed hyperbolic three manifold", Ann. of Math. (2) 175:3 (2012), 1127-1190. MR 2912704 Zbl 1254.57014

[Kassabov and Matucci 2011] M. Kassabov and F. Matucci, "Bounding the residual finiteness of free groups", Proc. Amer. Math. Soc. 139:7 (2011), 2281-2286. MR 2784792 Zbl 1230.20045

[Kharlampovich et al. 2014] O. Kharlampovich, A. Myasnikov, and P. Weil, "Stallings graphs for quasi-convex subgroups", preprint, 2014. arXiv 1408.1917

[Kozma and Thom 2016] G. Kozma and A. Thom, "Divisibility and laws in finite simple groups", Math. Ann. 364:1-2 (2016), 79-95. MR 3451381 Zbl 06540649

[McKinsey 1943] J. C. C. McKinsey, "The decision problem for some classes of sentences without quantifiers”, J. Symbolic Logic 8 (1943), 61-76. MR 0008991 Zbl 0063.03864

[Mostowski 1966] A. W. Mostowski, "On the decidability of some problems in special classes of groups”, Fund. Math. 59 (1966), 123-135. MR 0224693 Zbl 0143.03701

[Ollivier and Wise 2011] Y. Ollivier and D. T. Wise, "Cubulating random groups at density less than 1/6”, Trans. Amer. Math. Soc. 363:9 (2011), 4701-4733. MR 2806688 Zbl 1277.20048

[Patel 2013] P. Patel, Quantifying algebraic properties of surface groups and 3-manifold groups, thesis, Rutgers University, New Brunswick, NJ, 2013. MR 3192999

[Patel 2014] P. Patel, "On a theorem of Peter Scott”, Proc. Amer. Math. Soc. 142:8 (2014), 2891-2906. MR 3209342 Zbl 1311.57003

[Rivin 2012] I. Rivin, "Geodesics with one self-intersection, and other stories", Adv. Math. 231:5 (2012), 2391-2412. MR 2970452 Zbl 1257.57024

[Sageev and Wise 2015] M. Sageev and D. T. Wise, "Cores for quasiconvex actions", Proc. Amer. Math. Soc. 143:7 (2015), 2731-2741. MR 3336599 Zbl 06428953

[Scott 1978] P. Scott, "Subgroups of surface groups are almost geometric", J. Lond. Math. Soc. (2) 17:3 (1978), 555-565. MR 0494062 Zbl 0412.57006

[Wise 2004] D. T. Wise, "Cubulating small cancellation groups", Geom. Funct. Anal. 14:1 (2004), 150-214. MR 2053602 Zbl 1071.20038

[Wise 2011] D. T. Wise, “The structure of groups with a quasiconvex hierarchy", preprint, 2011, available at https://drive.google.com/file/d/0B45cNx80t5-2T0twUDFxVXRnQnc/view.

[Wise 2012] D. T. Wise, From riches to raags: 3-manifolds, right-angled Artin groups, and cubical geometry (New York, NY, 2011), CBMS Regional Conference Series in Mathematics 117, American Mathematical Society, Providence, RI, 2012. MR 2986461 Zbl 1278.20055

Received August 7, 2015. Revised September 29, 2015. 
MARK F. HAGEN

Department of Pure Mathematicss and Mathematical Statistics UNIVERSITY OF CAMBRIDGE

WILBERFORCE RD.

CAMBRIDGE

CB3 0WB

UNITED KINGDOM

markfhagen@gmail.com

PRIYAM PATEL

DEPARTMENT OF MATHEMATICS

PURDUE UNIVERSITY

150 N. UNIVERSITY ST.

WEST LAFAYETTE, IN 47907

UNITED STATES

pate1376@purdue.edu 


\title{
PACIFIC JOURNAL OF MATHEMATICS
}

Founded in 1951 by E. F. Beckenbach (1906-1982) and F. Wolf (1904-1989)

$$
\text { msp.org/pjm }
$$

\section{EDITORS}

\author{
Don Blasius (Managing Editor) \\ Department of Mathematics \\ University of California \\ Los Angeles, CA 90095-1555 \\ blasius@math.ucla.edu
}

\author{
Paul Balmer \\ Department of Mathematics \\ University of California \\ Los Angeles, CA 90095-1555 \\ balmer@math.ucla.edu \\ Robert Finn \\ Department of Mathematics \\ Stanford University \\ Stanford, CA 94305-2125 \\ finn@math.stanford.edu \\ Sorin Popa \\ Department of Mathematics \\ University of California \\ Los Angeles, CA 90095-1555 \\ popa@math.ucla.edu
}

\author{
Vyjayanthi Chari \\ Department of Mathematics \\ University of California \\ Riverside, CA 92521-0135 \\ chari@math.ucr.edu \\ Kefeng Liu \\ Department of Mathematics \\ University of California \\ Los Angeles, CA 90095-1555 \\ liu@math.ucla.edu \\ Igor Pak \\ Department of Mathematics \\ University of California \\ Los Angeles, CA 90095-1555 \\ pak.pjm@gmail.com \\ Paul Yang \\ Department of Mathematics \\ Princeton University \\ Princeton NJ 08544-1000 \\ yang@math.princeton.edu
}

\section{PRODUCTION}

Silvio Levy, Scientific Editor, production@msp.org

\section{SUPPORTING INSTITUTIONS}

ACADEMIA SINICA, TAIPEI

CALIFORNIA INST. OF TECHNOLOGY

STANFORD UNIVERSITY

UNIV. OF BRITISH COLUMBIA

UNIV. OF CALIFORNIA, BERKELEY

UNIV. OF CALIFORNIA, DAVIS

UNIV. OF CALIFORNIA, LOS ANGELES

UNIV. OF CALIFORNIA, RIVERSIDE

UNIV. OF CALIFORNIA, SAN DIEGO

UNIV. OF CALIF., SANTA BARBARA
KEIO UNIVERSITY

MATH. SCIENCES RESEARCH INSTITUTE

NEW MEXICO STATE UNIV.

OREGON STATE UNIV.
Daryl Cooper

Department of Mathematics

University of California

Santa Barbara, CA 93106-3080 cooper@math.ucsb.edu

Jiang-Hua Lu

Department of Mathematics

The University of Hong Kong

Pokfulam Rd., Hong Kong

jhlu@maths.hku.hk

$$
\text { Jie Qing }
$$

Department of Mathematics

University of California

Santa Cruz, CA 95064

qing@ cats.ucsc.edu

\author{
UNIV. OF CALIF., SANTA CRUZ \\ UNIV. OF MONTANA \\ UNIV. OF OREGON \\ UNIV. OF SOUTHERN CALIFORNIA \\ UNIV. OF UTAH \\ UNIV. OF WASHINGTON \\ WASHINGTON STATE UNIVERSITY
}

These supporting institutions contribute to the cost of publication of this Journal, but they are not owners or publishers and have no responsibility for its contents or policies.

See inside back cover or msp.org/pjm for submission instructions.

The subscription price for 2016 is US $\$ 440 /$ year for the electronic version, and \$600/year for print and electronic.

Subscriptions, requests for back issues and changes of subscriber address should be sent to Pacific Journal of Mathematics, P.O. Box 4163, Berkeley, CA 94704-0163, U.S.A. The Pacific Journal of Mathematics is indexed by Mathematical Reviews, Zentralblatt MATH, PASCAL CNRS Index, Referativnyi Zhurnal, Current Mathematical Publications and Web of Knowledge (Science Citation Index).

The Pacific Journal of Mathematics (ISSN 0030-8730) at the University of California, c/o Department of Mathematics, 798 Evans Hall \#3840, Berkeley, CA 94720-3840, is published twelve times a year. Periodical rate postage paid at Berkeley, CA 94704, and additional mailing offices. POSTMASTER: send address changes to Pacific Journal of Mathematics, P.O. Box 4163, Berkeley, CA 94704-0163.

PJM peer review and production are managed by EditFLOW ${ }^{\circledR}$ from Mathematical Sciences Publishers.

PUBLISHED BY

\section{I. mathematical sciences publishers}

nonprofit scientific publishing

http://msp.org/

(C) 2016 Mathematical Sciences Publishers 


\section{PACIFIC JOURNAL OF MATHEMATICS}

Volume $284 \quad$ No. $1 \quad$ September 2016

Bitwist manifolds and two-bridge knots

JAmes W. CANNON, William J. Floyd, LEeR LAMbert,

WALTER R. PARry and Jessica S. PurCELL

Recognizing right-angled Coxeter groups using involutions

Charles Cunningham, Andy Eisenberg, Adam Piggott and KIM RUANE

On Yamabe-type problems on Riemannian manifolds with boundary

Marco Ghimenti, Anna Maria Micheletti and Angela

PISTOIA

Quantifying separability in virtually special groups

MARK F. HAGEN and PRIYAM PATEL

Conformal designs and minimal conformal weight spaces of vertex operator superalgebras

TOMONORI HASHIKAWA

Coaction functors

S. KALiszewski, Magnus B. LANDSTAD and John QuigG

Cohomology and extensions of braces

VICTORIA LEBED and LEANDRO VENDRAMIN

Noncommutative differentials on Poisson-Lie groups and pre-Lie algebras

SHAHN MAJID and WEN-QING TAO 